\title{
Síndrome da Veia Cava Cranial em cão da raça Golden Retriever - Relato de caso
}

\author{
Cranial Vena Cava Syndrome in a Golden Retriever dog - Case report \\ Síndrome de la Vena Cava Craneal en un perro Golden Retriever - Reporte de caso
}

Recebido: 19/07/2021 | Revisado: 25/07/2021 | Aceito: 27/07/2021 | Publicado: 10/08/2021

\author{
Liege Vieira da Rosa Garcia \\ ORCID: https://orcid.org/0000-0002-8651-7523 \\ Universidade de Vassouras, Brasil \\ E-mail: vieiraliege@ outlook.com \\ Indira Ormond Machado Guimarães dos Santos \\ ORCID: https://orcid.org/0000-0003-1811-8077 \\ Médica Veterinária autônoma, Brasil \\ E-mail: indyormond@hotmail.com \\ Eduardo Butturini de Carvalho \\ ORCID: https://orcid.org/0000-0002-6411-1215 \\ Universidade de Vassouras, Brasil \\ Instituto de Biofísica Carlos Chagas Filho, Brasil \\ E-mail: butturini@gmail.com \\ Suzana Martins Gomes Leite \\ ORCID: https://orcid.org/0000-0002-8209-2038 \\ Médica Veterinária autônoma, Brasil \\ E-mail: suvetleite@gmail.com \\ Dianna Caroline Saiki \\ ORCID: https://orcid.org/0000-0003-1561-8754 \\ Universidade Federal Rural do Rio de Janeiro, Brasil \\ E-mail: diannacsaiki@gmail.com \\ Thiago Ravache Sobreira Leite \\ ORCID: https://orcid.org/0000-0002-5584-8231 \\ Médico Veterinário autônomo, Brasil \\ E-mail: thiagoravachesl@gmail.com \\ Renata Fernandes Ferreira de Moraes \\ ORCID: https://orcid.org/0000-0001-7129-1587 \\ Universidade de Vassouras, Brasil \\ E-mail: referreirauff@yahoo.com.br
}

\begin{abstract}
Resumo
A síndrome da veia cava cranial (SVCC) é a expressão clínica da obstrução do fluxo sanguíneo deste vaso, provocando a redução do fluxo do retorno venoso em direção ao átrio direito. Os sinais clínicos são inespecíficos, e variam entre dispneia, tosse, cianose, disfagia, edema de face, taquicardia, dilatação das veias do pescoço, caquexia, sons cardíacos abafados, silêncio pulmonar e engorgitamento das veias jugulares e da parede do tórax. O presente trabalho relata o caso de SVCC em um cão da raça Golden Retriever macho de seis anos, em decorrência de um tumor na base do coração, no átrio direito. Em seu primeiro atendimento na emergência clínica o animal apresentou dores à palpação abdominal, mucosas congestas, edema facial, perda de massa muscular no crânio, hipofonese cardíaca e ausculta pulmonar crepitante. Tendo como aporte os exames realizados no paciente, como hemograma, bioquímica, ecocardiograma, radiografia torácica e análise citológica da efusão pericárdica, foi definhada a conduta terapêutica para que o quadro clínico fosse amenizado. O animal veio a óbito 18 dias após o início do seu tratamento. Com a necropsia foi possível concluir o diagnóstico, e classificar o tumor como hemangiossarcoma, uma neoplasia maligna e agressiva, que se origina das células do endotélio vascular, e é responsável por uma alta taxa de mortalidade em cães, principalmente nas raças Pastor Alemão e Golden Retriever. Quando presente no coração, é comum que o hemangiossarcoma se desenvolva no átrio direito.
\end{abstract}

Palavras-chave: Cão; Coração; Hemangiossarcoma; Síndrome; Veia cava.

\begin{abstract}
The cranial vena cava syndrome (CVCS) is the physical manifestation of the obstruction of the blood flow of this vessel, causing a reduction in the flow of venous return towards the right atrium. Its clinical symptoms are unspecific, and range from dyspnea, cough, cyanosis, dysphagia, edema of the face, tachycardia, neck veins dilation, cachexia, muffled heart sounds, pulmonary silence, and engorgement of the jugular veins and thorax. This paper reports the case of CVCS in a male six-year-old Golden Retriever dog, as a result of a tumor in the base of the heart, in the right atrium. During his first visit to the emergency clinic, the animal presented pain to abdominal palpation, congestion of the mucous membranes, facial edema, skull muscle loss, cardiac hypophonesis, and crackling pulmonary auscultation.
\end{abstract}


Having as support the exams that were performed in the patient, as hemogram, biochemical, echocardiogram, chest Xray and pericardial effusion cytological analysis, it was defined the therapeutic conduct so that the clinical picture was softened. The animal died 18 days after the beginning of its treatment. The necropsy allowed us to conclude the diagnosis, and the tumor was classified as hemangiosarcoma, a malignant and aggressive neoplasm that originates from vascular endothelium cells, and it is considered responsible for a high mortality rate in dogs, especially in the German Shepherd and Golden Retriever breeds. When it is located in the heart, it is common for hemangiosarcoma to grow in the right atrium.

Keywords: Dog; Heart; Hemangiosarcoma; Syndrome; Vena cava.

\section{Resumen}

El síndrome de la vena cava craneal (SVCC) es la expresión clínica de la obstrucción del flujo sanguíneo de este vaso, causando una reducción en el flujo de retorno venoso hacia la aurícula derecha. Los signos clínicos son inespecíficos y varían entre disnea, tos, cianosis, disfagia, edema facial, taquicardia, dilatación de las venas del cuello, caquexia, ruidos cardíacos amortiguados, silencio pulmonar e ingurgitación de las venas yugulares y de la pared torácica. El presente trabajo reporta el caso de SVCC en un perro Golden Retriever macho de seis años, debido a un tumor en la base del corazón, en la aurícula derecha. En su primera visita a la emergencia clínica, el animal presentó dolor a la palpación abdominal, mucosas congestionadas, edema facial, pérdida de masa muscular en el cráneo, hipofonía cardíaca y auscultación pulmonar crepitante. Con base en las exploraciones realizadas en el paciente, como hemograma, bioquímica, ecocardiograma, radiografía de tórax y análisis citológico del derrame pericárdico, se redujo el abordaje terapéutico para que se pudiera reducir el cuadro clínico. El animal murió 18 días después del inicio de su tratamiento. Con la necropsia se pudo concluir el diagnóstico y clasificar el tumor como hemangiosarcoma, una neoplasia maligna y agresiva, que se origina en las células del endotelio vascular y es responsable de una alta tasa de mortalidad en perros, principalmente en las razas Pastor Alemán y Golden Retriever. Cuando está presente en el corazón, es común que el hemangiosarcoma se desarrolle en la aurícula derecha.

Palabras clave: Perro; Corazón; Hemangiosarcoma; Syndrome; Vena cava.

\section{Introdução}

A síndrome da veia cava cranial (SVCC) representa um conjunto de sinais e sintomas que são consequências da obstrução do fluxo sanguíneo através da veia cava cranial em direção ao átrio direito. Os sinais variam entre edema dos membros craniais, cianose e dilatação das veias do pescoço, sendo os mais comuns: dispneia, disfagia, cefaleia, tosse, edema dos membros craniais e ortopneia. Aproximadamente $73 \%$ a $97 \%$ dos casos de SVCC ocorrem durante a evolução de processos malignos intratorácicos por extensão ou compressão da veia cava cranial pelo próprio tumor ou por linfonodos mediastinais acometidos. (Lochridge et al., 1979; Parish et al., 1981; Cirino et al., 2005).

A veia cava cranial é formada pela união de duas veias braquiocefálicas inominadas, direita e esquerda, e está localizada no mediastino médio, à direita da artéria aorta e anteriormente à traqueia. A obstrução da veia cava pode ser causada pela compressão extrínseca do vaso, trombose, invasão tumoral ou por dificuldade do retorno venoso ao coração secundária a doenças intra-atriais ou intraluminais (Cirino et al., 2005).

Na medicina veterinária a SVCC é rara, e pode estar associada a algumas doenças, dentre elas obstrução trombótica e/ou fibrótica do átrio direito (Ferreira et al., 2010; Henz et al., 2019). As neoplasias cardíacas em sua grande maioria se originam no lado direito do coração, principalmente no átrio direito. Os tipos histológicos mais comuns na espécie canina são hemangiossarcoma, neoplasias de corpo aórtico, linfomas e tumores ectópicos de tireoide (Daleck \& De Nardi, 2016; Henz et al., 2019).

O presente estudo tem o objetivo de relatar o caso de um cão da raça Golden Retriever de seis anos de idade com SVCC causada por um hemangiossarcoma em átrio direito.

\section{Metodologia}

Este estudo foi conduzido a partir da assinatura do Termo de Consentimento Livre e Esclarecido (TCLE) pela tutora do animal autorizando o uso e disponibilizando fotos, vídeos e resultados de exames para o relato do caso clínico analítico e 
descritivo do fenômeno observado (Pereira et al., 2018), que foi construído através de um levantamento bibliográfico a respeito da casuística de hemangiossarcomas em cães e da síndrome da veia cava cranial.

\section{Relato de Caso}

Um cão da raça Golden Retriever de seis anos de idade, pesando 38,5kg foi atendido no setor de emergência de uma clínica veterinária no município do Rio de Janeiro no dia 21 de outubro de 2020, com queixa de edema de face (figura 1) e respiração ofegante, e o histórico relatado pelo tutor foi que, na semana anterior, o animal havia sido vermifugado com praziquantel $(5 \mathrm{mg} / \mathrm{kg})$, pamoato de pirantel $(14,4 \mathrm{mg} / \mathrm{kg})$ e febantel $(15 \mathrm{mg} / \mathrm{kg})$, apresentando diarreia logo após o uso.

Ao exame clínico o animal apresentava dores à palpação abdominal, mucosas congestas, edema facial, perda de massa muscular no crânio, hipofonese cardíaca, ausculta pulmonar crepitante e tempo de preenchimento capilar de dois segundos. Foram solicitados hemograma completo, bioquímica, urinálise e hemogasometria.

Ao longo da internação, foi realizada a sondagem vesical em circuito fechado, e o cão foi mantido no oxigênio através da cânula, e com acesso venoso para que fossem realizadas as medicações. Foi aplicada dexametasona ( $1 \mathrm{mg} / \mathrm{kg})$, prometazina $(0,3 \mathrm{mg} / \mathrm{kg})$ e furosemida $(2 \mathrm{mg} / \mathrm{kg})$ via intravenosa (IV).

O padrão respiratório se manteve comprometido e sua saturação de oxigênio não ultrapassou $85 \%$. Foi feita a pericardiocentese para tratar o tamponamento cardíaco e evitar consequente óbito, e o líquido coletado foi enviado para análise laboratorial. Durante o primeiro dia de internação o animal não defecou nem apresentou vômito.

Em relação às medicações, foram feitas também aplicações de metronidazol $(15 \mathrm{~m} / \mathrm{kg})$, dipirona $(25 \mathrm{mg} / \mathrm{kg})$, citrato de maropitant $(1,0 \mathrm{mg} / \mathrm{kg})$, omeprazol $(1 \mathrm{mg} / \mathrm{kg})$ via oral (VO) e ondansetrona $(1 \mathrm{mg} / \mathrm{kg}) \mathrm{IV}$, além do uso de prednisolona $(1 \mathrm{mg} / \mathrm{kg})$ e probiótico $(2 \mathrm{~g} / \mathrm{kg}) \mathrm{VO}$.

O resultado da urinálise não apresentou nenhuma alteração significativa, exceto hematúria sem proteinúria associada. Em relação à bioquímica sérica, a ureia e a creatinina se encontravam aumentadas 191,0mg/dL e 1,8mg/dL respectivamente (valor de referência para a ureia: $21,0-60,0 \mathrm{mg} / \mathrm{dL}$; valor de referência para a creatinina $0,5-1,4 \mathrm{mg} / \mathrm{dL}$ ) e o hemograma apresentou neutrofilia com linfopenia e discreta trombocitopenia. De acordo com o resultado da análise, a efusão pericárdica foi classificada como transudato modificado. A hemogasometria foi feita utilizando analisador sanguíneo portátil (i-STAT ${ }^{\circledR}$ ), com sangue arterial, temperatura de $37,7^{\circ} \mathrm{C}$ e o $\mathrm{pH} 7,35, \mathrm{PCO}_{2} 20,4 \mathrm{mmHg}, \mathrm{HCO}_{3} 11,4 \mathrm{mmol} / \mathrm{L}$ e excesso de bases (EB) $14 \mathrm{mmol} / \mathrm{L}$ caracterizando acidose metabólica e alcalose respiratória compensatória.

Foi realizada a avaliação ecocardiográfica (figura 2), e para isso o paciente foi sedado com aplicação do citrato de fentanil $(3 \mu / \mathrm{kg}) \mathrm{IV}$, seguido da administração de propofol $(2 \mathrm{mg} / \mathrm{kg}) \mathrm{IV}$. Foram reaplicadas doses de $1 \mathrm{mg} / \mathrm{kg}$ de propofol IV a cada 8 a 10 minutos, de acordo com a monitorização da oximetria de pulso e pressão arterial não invasiva, com objetivo de manter o estado de sedação. Durante o exame, foi ofertado oxigênio suplementar, por meio de sonda nasal unilateral e fluxo de $3 \mathrm{~L} / \mathrm{min}$. Na avaliação foi detectada presença de líquido no pericárdio e na pleura.

Ao segundo dia de internação o cão permaneceu prostrado, em decúbito esternal, sem tentar se levantar em nenhum momento. Esteve responsivo à manipulação, mantendo o padrão respiratório dentro do esperado, com melhora evidente no edema facial. $\mathrm{O}$ animal comeu com apetite tanto ração seca quanto a úmida; foi feita a reposição de bicarbonato ( $98 \mathrm{~mL}$ de bicarbonato a 8,4\% diluído em soro glicosado 5\% durante 6 horas) sem que houvesse nenhuma intercorrência. Foi mantido com cânula nasal para oxigenioterapia. Neste segundo dia o cão não defecou, não apresentou êmese nem sialorreia, mucosa normocorada, normoglicêmico, normotérmico, normotenso e com hipofonese cardíaca. 
O ecocardiograma realizado revelou um tumor na base do coração, no átrio direito. A medicação prescrita foi: pimobendan $(0,25 \mathrm{mg} / \mathrm{kg}$ ) BID (bis in die, em português duas vezes por dia) VO e furosemida (2mg/kg) SID (semel in die, em português, uma vez por dia) IV. O prognóstico se mantinha de reservado a desfavorável.

Já no terceiro dia de internação o animal continuou prostrado e em decúbito esternal, sendo mantido no suporte de oxigênio, com padrão respiratório um pouco forçado. $\mathrm{O}$ edema de face piorou novamente, sendo necessário aumentar a frequência da furosemida de SID para BID. O cão permaneceu sondado em circuito fechado, com urina concentrada, sem defecar, não apresentou êmese ou sialorreia; alimentando-se com apetite e mantendo boa ingesta hídrica, mucosas normocoradas, normotérmico, normotenso, normoglicêmico e hipofonese cardíaca.

No início do quarto dia de internação foi levado para caminhar. Apresentou dificuldade de locomoção, dor, diminuição da propriocepção em membros posteriores, mas conseguia se manter em estação, expondo muito cansaço e leve taquipneia. Apresentou polidipsia e normofagia, e estava responsivo à manipulação. Não houve necessidade de oxigenoterapia, porém o animal apresentou piora no quadro do edema de face e na coloração da urina. Não houve êmese nem sialorreia, e defecou uma vez. Mantinha as mucosas normocoradas, normotérmico, normotenso, normoglicêmico, taquipneico, tempo de preenchimento capilar de dois segundos e hipofonese. Foi feita radiografia do tórax que demostrou presença de líquido (figura 3).

Ao final do dia 24 de outubro de 2020, o animal teve alta e a medicação passou a ser realizada de forma oral. Doze dias depois, no dia 05 de novembro de 2020, ele retornou à clínica dispneico, pesando 34,4kg. Foi necessária oxigenoterapia com cânula nasal para que o seu padrão respiratório melhorasse. Havia grande quantidade de líquido em tórax que foi drenado (420ml aproximadamente), de coloração avermelhada (figura 4). O líquido foi encaminhado para análise. O cão apresentou-se prostrado, normotérmico, hipotenso e com as mucosas normocoradas, não se alimentou e apenas bebeu água.

No dia seguinte à sua segunda internação, permaneceu prostrado, sem tentar se movimentar, porém alerta, expressando reação ao se aproximar dele. O animal não defecou, não apresentou êmese e não comeu, apenas apresentou polidipsia. Estava levemente hipotérmico, normoglicêmico, hipotenso e com as mucosas normocoradas. Foi feita a coleta de sangue para realização do hemograma, e foi drenado mais de 1 litro de fluido do tórax. Foram feitas medicações IV: furosemida $(2 \mathrm{mg} / \mathrm{kg})$, dipirona $(25 \mathrm{mg} / \mathrm{kg})$ e ondansetrona $(1 \mathrm{mg} / \mathrm{kg})$, pimodendam $(0,25 \mathrm{mg} / \mathrm{kg}) \mathrm{VO}$, e o animal estava em fluidoterapia $(40 \mathrm{ml} / \mathrm{kg} / \mathrm{dia})$. O laudo da análise citológica do líquido coletado do tórax no dia anterior foi descrito como transudato modificado.

O hemograma apresentou leucocitose com neutrofilia, monocitose e eosinopenia absolutas, e trombocitopenia. A bioquímica sérica não apresentou muitas alterações, exceto ureia (170mg/dL) e creatinina (3,8mg/dL) aumentadas, hiponatremia (139,0 mmol/L sódio) e hipercalemia (7,2 mmol/L potássio). Valor de referência para o sódio 141,0 - 152,0 $\mathrm{mmol} / \mathrm{L}$; valor de referência para o potássio $4,3-5,4 \mathrm{mmol} / \mathrm{L}$.

No dia 7 de novembro de 2020 o animal estava com o padrão respiratório restritivo com esforço abdominal. Foi feita furosemida $(2 \mathrm{mg} / \mathrm{kg}) \mathrm{VO}$, porém vomitou logo em seguida, optando-se pela administração do fármaco de forma IV. O paciente apresentou dor à palpação, e foi administrado então tramadol $(2 \mathrm{mg} / \mathrm{kg}$ ) e dipirona (25mg/kg) TID (ter in die, em português, três vezes por dia). Devido ao vômito, foi passado ondansetrona para TID também. Ao longo do plantão o padrão respiratório piorou (redução dos ruídos auscultatórios do lado esquerdo), e a oximetria baixou para 75\%. Foi feito aporte de oxigênio e sedação (metadona $0,2 \mathrm{mg} / \mathrm{kg}$ e midazolam $0,1 \mathrm{mg} / \mathrm{kg}$ ), mas o cão permaneceu com padrão respiratório insatisfatório. Algumas horas depois apresentou piora no quadro, entrou em angústia respiratória e veio a óbito.

Foi realizada a necropsia (figura 5) e o coração foi encaminhado para exame histopatológico. A conclusão foi hemangiossarcoma em átrio direito, grau histológico II. Na macroscopia o coração estava medindo em seus maiores eixos 11,0 x 7,3 x 5,6 cm. 
Em porção medial do átrio direito foi observado um nódulo irregular de 3,0 x 2,5 x 1,4 cm. Ao corte apresentou consistência firme e superfície compacta de tonalidade pardacenta a acastanhada. Em porção cranial do átrio direito foi observada presença de formação irregular de 5,0 x 3,2 x 2,6 cm. Ao corte apresentou consistência firme e superfície de corte compacta de tonalidade brancacenta com focos enegrecidos.

Na microscopia, o parênquima cardíaco revelou proliferação de origem mesenquimal, em arranjo sólido, por vezes esboçando fendas e cavitações vasculares de tamanhos variados. Células neoplásicas fusiformes, com citoplasma de bordos indistintos, núcleo alongado a ovalado e um a 2 pequenos nucléolos foram observados. Presença de moderado pleomorfismo nuclear. Uma a três figuras de mitose em aumento de 400x associadas a algumas células multinucleadas foram observadas. O parênquima restante apresentou moderada hemorragia, congestão e necrose, além de alguns trombos vasculares e extenso infiltrado inflamatório predominantemente supurado de permeio a massa neoplásica. Havia comprometimento da histoarquitetura do parênquima.

Figura 1. Paciente com edema de face.

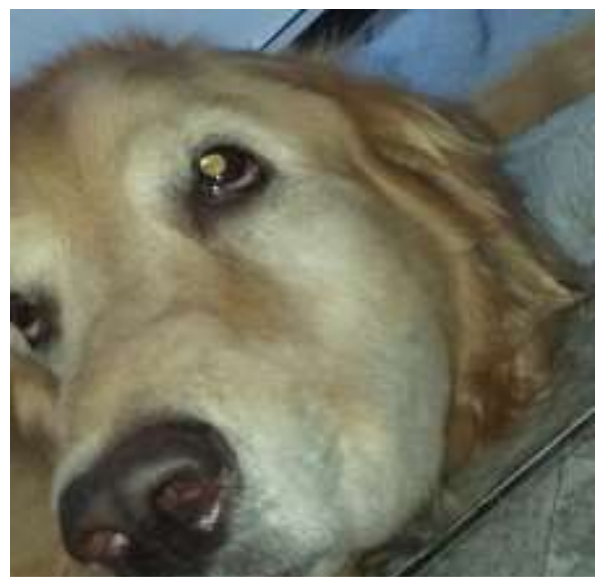

Figura 3. Radiografia que demonstra a presença de fissuras interlobares e lobos pulmonares em "folha", indicando efusão pleural, e parênquima pulmonar com aumento da densidade bronco-intersticial em campos pulmonares caudais, sugestivo para pneumopatia.

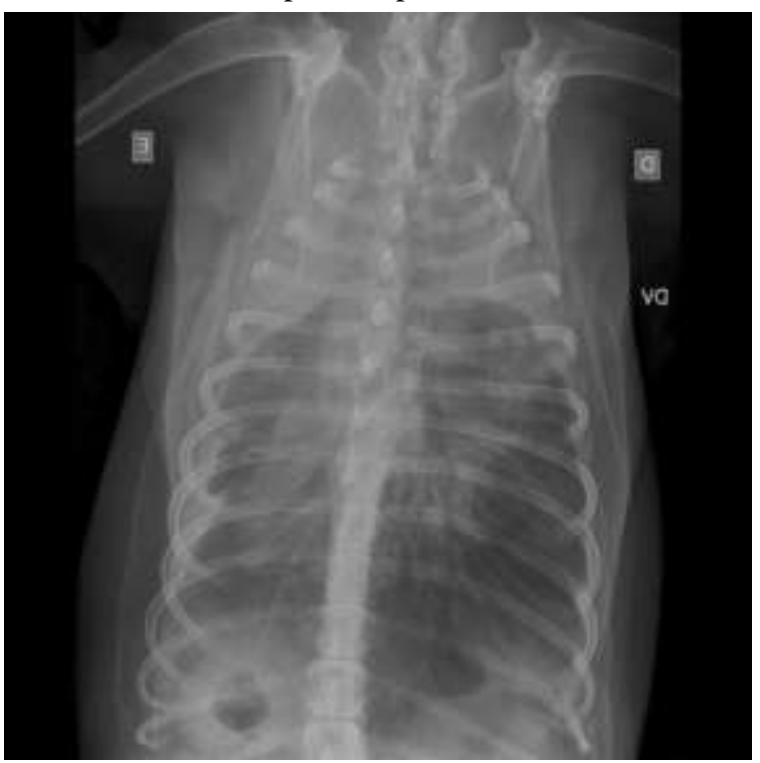

Figura 2. Ecocardiograma do paciente que demonstra o comprometimento de $95 \%$ do átrio direito.

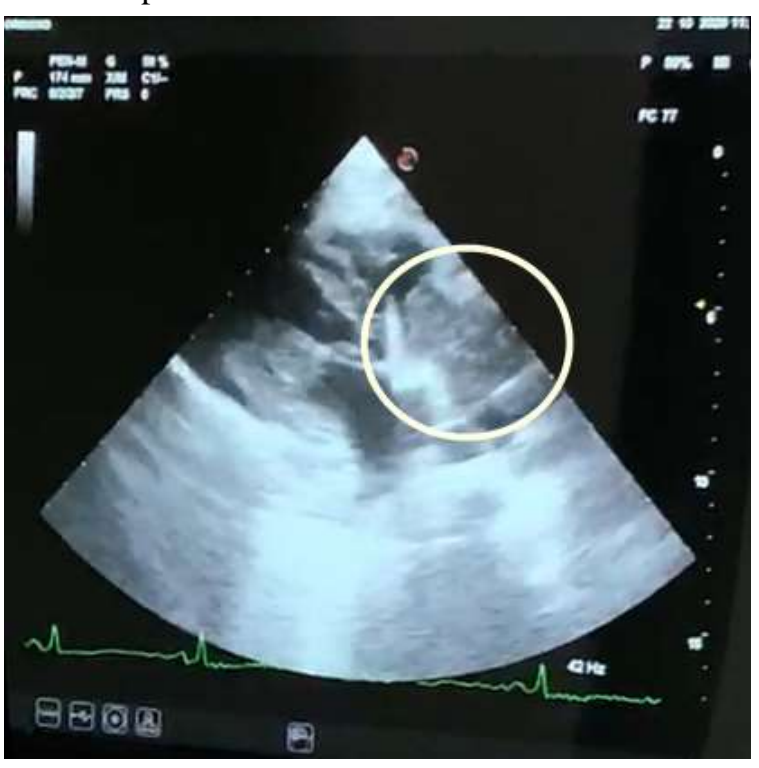

Figura 4. Drenagem de líquido sanguinolento do tórax classificado como transudato modificado.

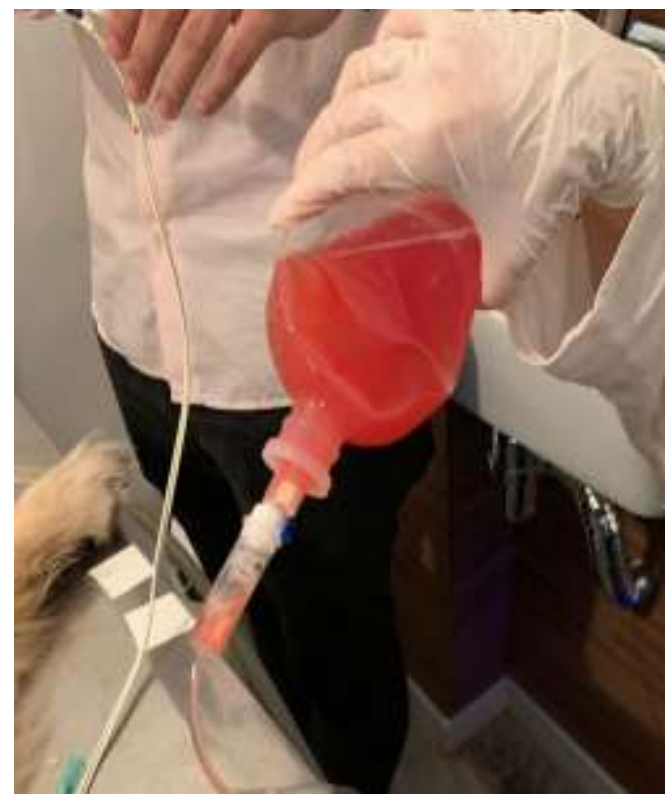

Fonte. Autores. 
Figura 5. Coração medindo em seus maiores eixos 11,0 x 7,3 x $5,6 \mathrm{~cm}$. Em porção medial do átrio direito, presença de nódulo irregular de 3,0 x 2,5 x 1,4 cm. Em porção cranial do átrio direito, presença de formação irregular de 5,0 x 3,2 x 2,6 $\mathrm{cm}$. Hemangiossarcoma, grau histológico II.

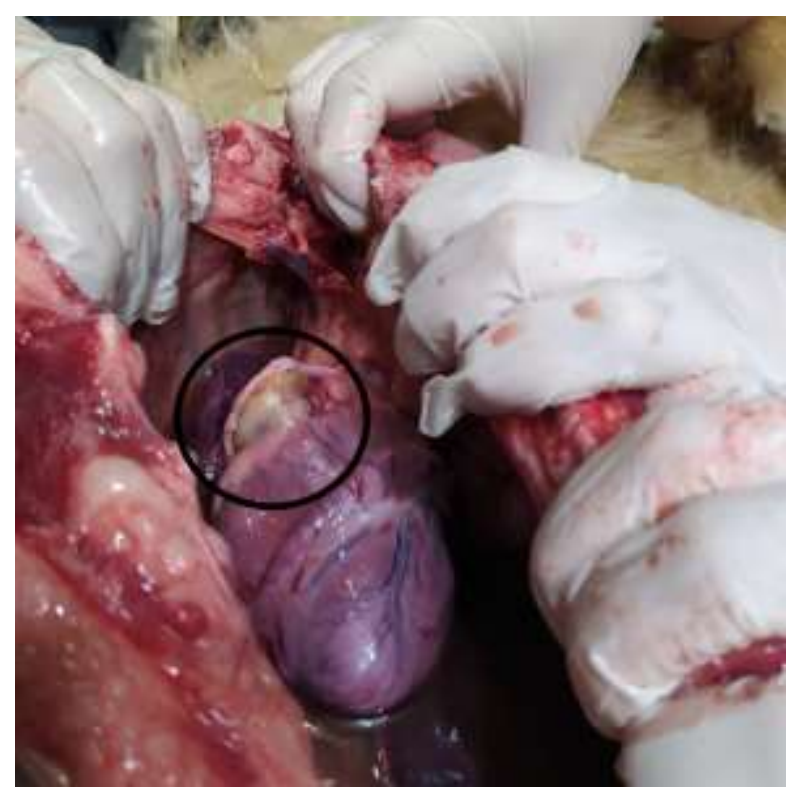

Fonte. Autores.

\section{Discussão}

Hemangiossarcoma é uma neoplasia maligna e agressiva, que se origina das células do endotélio vascular, e é responsável por uma alta taxa de mortalidade em cães, principalmente nas raças Pastor Alemão e Golden Retriever, sendo predominante nos machos entre 8 e 10 anos de idade (Nelson \& Couto, 2015; Daleck \& De Nardi, 2016; Henz et al., 2019).

O crescimento atípico e a proliferação celular desenfreada são características do tecido neoplásico, tendo este, crescimento mais acelerado do que os tecidos adjacentes normais. A magnitude das alterações cardiovasculares determinadas por esta neoplasia varia conforme a localização, tamanho, presença de efusão pericárdica e tamponamento cardíaco resultante. As neoplasias estão entre as principais causas de mortes de cães no Brasil (Fruchter et al., 1992; Ferreira et al., 2011; Martins, et al., 2011; Henz et al., 2019). Observadas em cães, as neoplasias ocorrem com mais frequência na cabeça, abdômen, membros anteriores, posteriores, períneo e dígitos, e a sua diversidade varia geograficamente em função da susceptibilidade individual, hábitos dos animais e aos fatores ambientais (Ribeiro et al., 2020; Corrêa et al., 2021).

Quando presente no coração, é comum que o hemangiossarcoma se desenvolva no átrio direito, em especial no apêndice auricular direito, ou nos tecidos adjacentes; contudo pode ser observado em outros locais, como junção atrioventricular direita, lúmen atrial e ventricular e ventrículo esquerdo. Metástases pulmonares são frequentes, bem como nos rins, baço e fígado, não sendo o caso deste paciente (Keene et al., 1990; Palacio et al., 2006; Ferreira et al., 2011).

Neves (2017) \& Henz et al. (2019) afirmam que a localização e o tamanho do tumor podem provocar a compressão da traqueia, brônquios e esôfago, e ocasionar sinais clínicos como tosse, dispneia, disfagia e regurgitação. O paciente deste relato apresentou dispneia e êmese, e por algumas vezes não teve apetite, podendo considerar que houve uma compressão por parte 
do hemangiossarcoma. Pinto (2015) \& Martins \& Gomes (2019) destacam que quando o nódulo se desenvolve no coração, o animal manifesta sinais clínicos como arritmias cardíacas, hemorragia do pericárdio, tamponamento cardíaco, insuficiência cardíaca direita (ICD) e dificuldade respiratória; exceto a ICD, o paciente apresentou todos esses sinais relacionados.

No caso relatado, por conta da localização do tumor e consequente acúmulo de líquido em pericárdio, o cão apresentou um quadro de tamponamento cardíaco, que foi estabilizado após pericardiocentese, procedimento indicado nesses casos. Tobias (2005) \& Salinas et al. (2017) afirmam que para tratar a efusão pericárdica e o tamponamento cardíaco é necessário realizar a pericardiocentese, para reduzir a pressão intrapericárdica e restaurar a hemodinâmica do sistema cardiovascular. Quando há derrame pericárdico, recomenda-se a pericardiocentese terapêutica e diagnóstica durante o exame ecocardiográfico (Neves, 2017; Henz et al., 2019).

Os cães que apresentam hemangiossarcoma cardíaco são capazes de acumular efusão pericárdica e colapso, proveniente de tamponamento cardíaco agudo, que se caracterizam por uma menor quantidade de líquido acumulado, enquanto animais com efusão pericárdica secundária a outras causas, que não tumor cardíaco, acumulam de forma lenta maiores quantidades de líquido (cerca de 730ml) e apresentam pequenas alterações hemodinâmicas (Johson et al., 2004; Ferreira et al., 2011). Na primeira coleta, o paciente se encontrava com pouco líquido no pericárdio e grande quantidade no tórax, um volume de aproximadamente $420 \mathrm{ml}$ com coloração avermelhada. Já na segunda, foi drenado mais de 1 litro de fluido do tórax.

Sempre que o paciente apresentar um quadro crítico, é necessário realizar exames complementares para apoio ao diagnóstico como hemograma, bioquímica, eletrocardiograma e análise citológica de derrames pleurais e pericárdicos quando presentes, visto que os sinais clínicos são inespecíficos em grande parte dos casos, o que muitas vezes ocasiona um diagnóstico tardio, quando o tumor já alcançou um tamanho razoável (Kim et al, 2015; Neves, 2017; Henz et al., 2019; Martins \& Gomes, 2019). Neste caso, foram feitos hemograma, bioquímica, ecocardiografia, radiografia e análise citológica do derrame pericárdico e torácico, sendo o exame ecocardiográfico imprescindível para localização e sugestão de neoplasia, visto que todos os exames complementares que foram realizados não tiveram resultados conclusivos, incluindo a análise das efusões pericárdicas e pleurais que foram classificadas como transudato modificado.

Líquidos classificados como transudato modificado são aqueles que contêm proteínas e células em sua composição. O líquido drenado do tórax do paciente apresentava aspecto hemorrágico, concordando com Nelson \& Couto (2015) \& Henz et al. (2019), que citam este tipo de derrame como o mais comum em cães, e geralmente com origem neoplásica ou idiopática. Nos líquidos hemorrágicos há pincipalmente presença de eritrócitos, mas pode haver também células neoplásicas e mesoteliais. Cabe salientar que a análise citológica não é o principal exame para diagnosticar neoplasia cardíaca e outros tumores devido à sua baixa capacidade de esfoliar células neoplásicas, porém serve como uma tentativa de identificar o tipo histológico do tumor (Cagle et al., 2014; Salinas et al., 2017).

Logo na primeira internação do paciente, foi aplicada dexametasona $1 \mathrm{mg} / \mathrm{kg}$, anti-inflamatório esteroidal, e prometazina $0,3 \mathrm{mg} / \mathrm{kg}$, anti-histamínico $\mathrm{H} 1$ pertencente ao grupo das fenotiazinas, para a suspeita inicial de que o cão estava em um quadro de reação alérgica a picada de inseto, uma vez que ele apresentava edema na face, fato que reitera a importância de se fazer todos os exames complementares para a condução do caso e adequação da medicação. A furosemida $2 \mathrm{mg} / \mathrm{kg}$ também foi utilizada como diurético, se tratando também de um medicamento usado para auxiliar na dissolução de edemas relacionados à intercorrências cardíacas, hepáticas ou renais (Manzi et al., 2011).

Também foram feitas aplicações de metronidazol $(15 \mathrm{mg} / \mathrm{kg})$, composto que possui atividade antibacteriana; dipirona $(25 \mathrm{mg} / \mathrm{kg})$, que é um analgésico, que foi utilizado pois o animal apresentava bastante dor à palpação abdominal; citrato de maropitant $(1,0 \mathrm{mg} / \mathrm{kg})$ e ondansetrona $(1 \mathrm{mg} / \mathrm{kg})$ que são antieméticos; omeprazol $(1 \mathrm{mg} / \mathrm{kg})$, que se trata de um protetor gástrico comumente utilizado quando o paciente está ingerindo grande quantidade de medicação, a fim de proteger a mucosa do estômago; todos via IV. 
Por VO foram administradas a prednisolona $(1 \mathrm{mg} / \mathrm{kg})$ anti-inflamatório e imunossupressor indicado para tratar algumas inflamações, dentre elas as relacionadas à respiração, justificando seu uso pelo quadro de dispneia apresentado pelo paciente; probiótico $(2 \mathrm{~g} / \mathrm{kg})$ para recompor a flora intestinal, visto que o animal apresentou algumas vezes dificuldade para defecar, e pimobendan $(0,25 \mathrm{mg} / \mathrm{kg})$, que é um sensibilizador de cálcio e inibidor seletivo da fosfodiesterase 3 com efeitos inotrópicos e vasodilatadores positivos, indicado para terapias relacionadas à insuficiência sistólica e vasoconstrição/aumento de pós-carga, como o paciente apresentava um comprometimento cardíaco, o fármaco foi eleito (Manzi et al., 2011).

No primeiro hemograma do paciente foi constatada, além de outros achados, discreta trombocitopenia e leucocitose com neutrofilia. A leucocitose é um achado comum em cães com hemangiossarcoma, e a trombocitopenia também, esta acontece devido à hemorragia crônica, podendo ocorrer em até 75\% dos casos (Daleck \& De Nardi, 2016; Neves, 2017; Henz et al., 2019).

Para realizar o ecocardiograma foi necessária a sedação do paciente para uma melhor avaliação do animal e evitar que

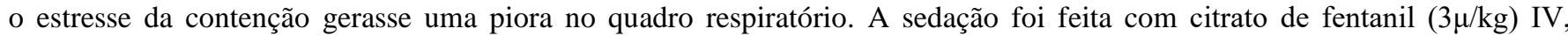
seguido da administração de propofol $(2 \mathrm{mg} / \mathrm{kg})$ IV. O fentanil foi utilizado com o intuito de promover sedoanalgesia e, assim, reduzir a dose necessária do propofol, diminuindo a ocorrência de efeitos cardiodepressores (Covey-Crump \& Murison, 2008).

A radiografia do tórax do paciente demostrava presença de fissuras interlobares e lobos pulmonares em "folha", indicando efusão pleural, e parênquima pulmonar com aumento da densidade bronco-intersticial em campos pulmonares caudais, sugestivo de pneumopatia, corroborando com Nelson \& Couto (2015) \& Henz et al. (2019), que afirmam que em cães com hemangiossarcoma é possível observar efusão pericárdica, efusão pleural, metástase pulmonar, veia cava caudal distendida e desvio traqueal.

Nos casos em que a efusão pericárdica é recorrente, o tratamento cirúrgico para o hemangiossarcoma cardíaco é uma opção, principalmente nos casos de neoplasias primárias, pequenas e bem definidas em apêndice auricular direito. Contudo, a viabilidade da ressecção de hemangiossarcoma depende de alguns fatores, como não envolver válvulas cardíacas, vasos coronários e/ou vida de saída do ventrículo direito (Daleck \& De Nardi, 2016; Neves, 2017; Henz et al., 2019). Apesar de ser possível a remoção cirúrgica do tumor em alguns casos, não era a circunstância deste paciente, que apresentava um comprometimento de $95 \%$ do átrio direito, sem ter como fazer a remoção do tumor com a devida margem de segurança, ele perderia o átrio, causando o óbito.

O cão deste estudo apresentava SVCC, que é a expressão clínica da obstrução do fluxo sanguíneo deste vaso, provocando a redução do fluxo do retorno venoso em direção ao átrio direito. Dos sinais clínicos da SVCC descritos por Ferreira et al. (2010), como cianose, dispneia, tosse, disfagia, dilatação das veias do pescoço, edema de face, edema na região cervical e porção cranial do tórax, além dos apêndices torácicos, caquexia, taquicardia, sons cardíacos abafados, silêncio pulmonar e engorgitamento das veias jugulares e da parede do tórax, alguns foram observados no paciente, sendo esses: edema de face e hipofonese cardíaca.

O paciente estava com SVCC causada por um hemangiossarcoma em átrio direito, e de acordo com Ferreira et al. (2010) \& Henz et al. (2019) na medicina veterinária esta síndrome é rara, e geralmente está relacionada à dirofilariose, obstrução trombótica e/ou fibrótica do átrio direito ou por compressão por processos malignos. Neste caso, o paciente apresentava um hemangiossarcoma no átrio direito, que culminou com a obstrução de parte do órgão em questão, o que confirma a afirmação dos autores.

A SVCC tem lenta progressão, variando de semanas a meses, associada a sintomas abruptos, que constituem uma emergência médica. Os pacientes com esta síndrome apresentam grande desconforto e podem desenvolver complicações que colocam sua vida em risco, como o edema de laringe ou cerebral (Hochrein et al., 1998; Ferreira et al., 2010). Neste animal o maior complicador foi o acúmulo de líquido em tórax que dificultou a respiração do animal, efusão pleural e pericárdica, 
seguida do edema.

Terapia com radiação e/ou quimioterapia geralmente são utilizadas para tratar os casos de SVCC causadas por neoplasias malignas, entretanto a resolução dos sintomas pode ocorrer lentamente ou de forma incompleta com estas terapias (Kirk, 1994; Ferreira et al., 2010). Neste caso, os responsáveis pelo animal optaram por não realizar a quimioterapia, uma vez que o diagnóstico ainda não era definitivo, ele só foi concluído post mortem, e quimioterápicos de forma geral têm efeitos colaterais agressivos.

Dahl et al. (2008) \& Salinas et al. (2017) acreditam, que a sobrevivência do paciente acometido com hemangiossarcoma está diretamente relacionada à sua idade no momento do diagnóstico, quanto mais avançada a idade, menor a expectativa de vida. O animal veio a óbito 18 dias após a primeira internação, quando foi retirado o coração para análise histopatológica, fechando o diagnóstico. O resultado do exame foi hemangiossarcoma grau II. De acordo com Never (2017) \& Henz et al. (2019), independente da conduta terapêutica adotada pelo médico veterinário, o prognóstico das neoplasias cardíacas varia de reservado a desfavorável dependendo do tipo histológico do tumor, sendo o do hemangiossarcoma bastante reservado.

\section{Considerações Finais}

A SVCC é rara em cães, por isso é de extrema importância uma análise criteriosa do histórico e anamnese do paciente, feitas pelo médico veterinário, associada à realização dos exames complementares, principalmente quando uma das causas é a compressão do átrio direito por tumores malignos. O diagnóstico de hemangiossarcoma muitas vezes não é possível de ser feito ainda com o animal em vida por conta da sua localização e tamanho, inviabilizando a excisão cirúrgica, e com isso o tratamento evolui de acordo com a clínica e os exames do paciente. A inviabilidade de confirmar o diagnóstico fez com que as terapias utilizadas tivessem o intuito de amenizar os sintomas e melhorar a qualidade da vida do paciente no tempo em que ainda restava para ele, visto que o prognóstico era desfavorável. Nos casos de SVCC a pericardiocentese é fundamental para trazer conforto ao animal, quantas vezes for necessária. O cão teve uma sobrevida de 18 dias, comprovando a agressividade do hemangiossarcoma e sua rápida progressão. Sendo assim, a avaliação histopatológica post mortem do átrio direito foi fundamental para concluir o caso relatado. Para melhor compreensão desta síndrome rara sugere-se a realização de estudos mais aprofundados na área da medicina veterinária

\section{Referências}

Cagle, L. A., Epstein, S. E., Owens, S. D., Mellema, M. S., Hopper, K., \& Burton, A. G. (2014). Diagnostic yield of cytologic analysis of pericardial effusion in dogs. Journal of veterinary internal medicine, 28(1), 66-71.

Cirino, L. M. I., Coelho, R. F., Rocha, I. D. D., \& Batista, B. P. D. S. N. (2005). Tratamento da síndrome da veia cava superior. Jornal Brasileiro de Pneumologia, 31(6), 540-550.

Corrêa, L. G., de Castro, C. C., da Silva, L. M. C., n Rossato, A. D. P., Berselli, M., Grecco, F. B., ... \& Fernandes, C. G. (2021). Fatores prognósticos e seu papel na classificação histológica dos carcinoma de células escamosas cutâneos. Research, Society and Development, 10(6), e52010615837-e52010615837. http://dx.doi.org/10.33448/rsd-v10i6.15837

Covey-Crump, G. L., \& Murison, P. J. (2008). Fentanyl or midazolam for co-induction of anaesthesia with propofol in dogs. Veterinary anaesthesia and analgesia, 35(6), 463-472.

Dahl, K., Gamlem, H., Tverdal, A., Glattre, E., \& Moe, L. (2008). Canine vascular neoplasia-a population-based study of prognosis. APMIS, 116, 55-62.

Daleck, C. R., \& De Nardi, A. B. (2016). Oncologia em cães e gatos. Grupo Gen-Editora Roca Ltda.

Neves, F. A. D. (2017). Estudo de tumores cardíacos caninos (Doctoral dissertation, Universidade de Lisboa, Faculdade de Medicina Veterinária).

Ferreira, A. R. A. (2011). Hemangiossarcoma cardíaco em cão: relato de caso. Medicina Veterinária (UFRPE), 17-25.

Ferreira, F. S., Silveira, L. L., Vale, D. F., Salavessa, C. M., Barretto, F. L., Oliveira, A. L., ... \& Carvalho, C. B. (2010). Síndrome da veia cava cranial (SVCC) secundária a quimiodectoma aórtico em cão-relato de caso Cranial vena cava syndrome secondary to aortic chemodectoma in dog. (UENFDR), 63-70 
Research, Society and Development, v. 10, n. 10, e10101018397, 2021

(CC BY 4.0) | ISSN 2525-3409 | DOI: http://dx.doi.org/10.33448/rsd-v10i10.18397

Fruchter, A. M., Miller, C. W., \& O'Grady, M. R. (1992). Echocardiographic results and clinical considerations in dogs with right atrial/auricular masses. The Canadian Veterinary Journal, 33(3), 171.

Girard, C., Helie, P., \& Odin, M. (1999). Intrapericardial neoplasia in dogs. Journal of Veterinary Diagnostic Investigation, 11(1), 73-78.

Henz, Y. F. (2019). Hemangiossarcoma cardíaco em cão da raça Golden Retriever: relato de caso.

Hochrein, J., Bashore, T. M., O’Laughlin, M. P., \& Harrison, J. K. (1998). Percutaneous stenting of superior vena cava syndrome: a case report and review of the literature. The American journal of medicine, 104(1), 78-84.

Keene, B. W., Rush, J. E., Cooley, A. J., \& Subramanian, R. (1990). Primary left ventricular hemangiosarcoma diagnosed by endomyocardial biopsy in a dog. Journal of the American Veterinary Medical Association, 197(11), 1501-1503.

Kim, M., Choi, S., Choi, H., Lee, Y., \& Lee, K. (2015). Diagnosis of a large splenic tumor in a dog: computed tomography versus magnetic resonance imaging. Journal of Veterinary Medical Science, 77(12), 1685-1687. https://doi.org/10.1292/jvms.15-0262

Kirk, R. W. (1994). Terapéutica veterinaria de pequeños animales (No. Sirsi) a372037).

Lochridge, S. K., Knibbe, W. P., \& Doty, D. B. (1979). Obstruction of the superior vena cava. Surgery, 85(1), 14-24.

Manzi, B. L.; Squariz, C. B.; Inouê, I. Y.; Piva, L. C.; Paschoal, P. (2011). Guia de medicamentos. Uniara-Centro Universitário de Araraquara. P 227.

Martins, D. B., Teixeira, L. V., França, R. T., \& Lopes, S. T. A. (2011). Biologia tumoral no cão: Uma revisão. Medvep-Revista Científica de Medicina Veterinária-Pequenos Animais e Animais de Estimação, 9(31), 630-637.

Martins, K. P. M., de Almeida, C. B., \& Gomes, D. E. (2019). Hemanfiossarcoma canino. Revista Científica, l(1).

Mesquita, L. P., Abreu, C. C., Nogueira, C. I., Wouters, A. T., Wouters, F., Bezerra Júnior, P. S., ... \& Varaschin, M. S. (2012). Prevalência e aspectos anatomopatológicos das neoplasias primárias do coração, de tecidos da base do coração e metastáticas, em cães do Sul de Minas Gerais (1994-2009). Pesquisa Veterinária Brasileira, 32(11), 1155-1163. https://doi.org/10.1590/S0100-736X2012001100014

Nelson, R., \& Couto, C. G. (2015). Medicina interna de pequenos animais. Elsevier Brasil.

Palacio, M. J. F. D., López, J. T., del Río, A. B., Alcaraz, J. S., Pallarés, F. J., \& Martinez, C. M. (2006). Left ventricular outflow tract obstruction secondary to hemangiosarcoma in a dog. Journal of veterinary internal medicine, 20(3), 687-690.

Parish, J. M., Marschke Jr, R. F., Dines, D. E., \& Lee, R. E. (1981, July). Etiologic considerations in superior vena cava syndrome. In Mayo Clinic Proceedings (Vol. 56, No. 7, pp. 407-413).

Pereira, A. S., Shitsuka, D. M., Parreira, F. J., \& Shitsuka, R. (2018). Metodologia da pesquisa científica.

Pinto, M. P. R. (2015). Hemangiossarcoma multicêntrico canino: relato de caso.

Ribeiro, T. A., Ferreira, V. R. F., Mondêgo-Oliveira, R., Andrade, F. H. E., Abreu-Silva, A. L., Oliveira, I. S., ... \& Torres, M. A. O. (2020). Epidemiological profile of canine neoplasms in São Luís/MA: a retrospective study (2008-2015). Research, Society and Development, 9(12), e8291210496-e8291210496. http://dx.doi.org/10.33448/rsd-v9i12. 10496

Salinas, E., Dávila, R., \& Chávez, E. (2017). Hemangiosarcoma Cardiaco Primario en Aurícula Derecha en un Canino Rottweiler de Ocho Años de Edad. Revista de Investigaciones Veterinarias del Perú, 28(4), 1039-1046. http://dx.doi.org/10.15381/rivep.v28i4.13875

Tobias, A. H. (2005). Pericardial disorders. In: Ettinger SJ, Feldman EC (eds). Textbook of veterinary internal medicine. 6th ed. St Louis, MO: Saunders. P $1107-1108$

Walter, J. H., \& Rudolph, R. (1996). Systemic, metastatic, eu-and heterotope tumours of the heart in necropsied dogs. Journal of Veterinary Medicine Series A, 43(1-10), 31-45. 